\title{
Aggregated $\alpha$-Synuclein Mediates Dopaminergic Neurotoxicity In Vivo
}

\author{
Magali Periquet, ${ }^{1}$ Tudor Fulga, ${ }^{1}$ Liisa Myllykangas, ${ }^{1}$ Michael G. Schlossmacher, ${ }^{2}$ and Mel B. Feany ${ }^{1}$ \\ ${ }^{1}$ Department of Pathology and ${ }^{2}$ Center for Neurologic Diseases, Department of Neurology, Brigham and Women's Hospital, Harvard Medical School, \\ Boston, Massachusetts 02115
}

\begin{abstract}
Mutations in the synaptic protein $\alpha$-synuclein cause rare genetic forms of Parkinson's disease. $\alpha$-Synuclein is thought to play a critical role in more common sporadic cases of Parkinson's disease as well because the protein aggregates in the hallmark intraneuronal inclusions of the disorder, Lewy bodies. To test the role of protein aggregation in the pathogenesis of Parkinson's disease, we expressed a form of $\alpha$-synuclein with a deletion of amino acids 71-82 that is unable to aggregate in vitro in a transgenic Drosophila model of the disorder. We found no evidence of large aggregates or oligomeric species of $\alpha$-synuclein in these animals and no loss of tyrosine hydroxylase-positive neurons. We also expressed a truncated form of $\alpha$-synuclein that has enhanced ability to aggregate in vitro. This truncated form of $\alpha$-synuclein showed increased aggregation into large inclusions bodies, increased accumulation of high molecular weight $\alpha$-synuclein species, and demonstrated enhanced neurotoxicity in vivo. Our findings thus support a critical role for aggregation of $\alpha$-synuclein in mediating toxicity to dopaminergic neurons in vivo, although the precise role each aggregated form of $\alpha$-synuclein plays in neurotoxicity remains to be determined.
\end{abstract}

Key words: Parkinson's; Drosophila; $\alpha$-synuclein; aggregation; dopaminergic; C-terminal truncation

\section{Introduction}

Parkinson's disease is a common neurodegenerative disorder affecting one in 50 individuals over the age of 60 (Moghal et al., 1994). Clinical manifestations reflect a relatively specific loss of dopaminergic neurons within the substantia nigra and include tremor, bradykinesia, and stiffness. The most specific histopathologic feature of Parkinson's disease is the Lewy body, a ubiquitinated proteinaceous inclusion present in the neuronal perikarya. Similar inclusions are also seen in neuronal processes where they are termed Lewy neurites (Cookson, 2005). $\alpha$-Synuclein appears to be a major component of both Lewy bodies and Lewy neurites, thus implicating $\alpha$-synuclein in the pathogenesis of Parkinson's disease (Cookson, 2005). In addition, missense mutations (Polymeropoulos et al., 1997; Kruger et al., 1998; Zarranz et al., 2004) and genomic duplication and triplication of the $\alpha$-synuclein gene (Singleton et al., 2003; Ibanez et al., 2004) cause autosomal dominant familial Parkinson's disease.

Given the prominence of protein aggregates in the pathology of Parkinson's disease, a number of studies have investigated the amino acids of $\alpha$-synuclein critical for aggregation in vitro. The

Received June 1, 2006; accepted Feb. 18, 2007.

This work was supported by the American Parkinson Disease Association (M.P.), National Institutes of Health Grants NS041536 (M.B.F.) and NS038375-07 (M.G.S.), the Lefler Foundation (M.P.), and the Finnish Parkinson Association (L.M.). Fly stocks were obtained from the Bloomington Stock Center. We thank Douglas Rennie at the Cutaneous Biology Research Center at Massachusetts General Hospital for expert assistance with transgenic fly production.

Correspondence should be addressed to Mel B. Feany, Department of Pathology, New Research Building, Room 630, 77 Avenue Louis Pasteur, Boston, MA 02115. E-mail: mel_feany@hms.harvard.edu.

Liisa Myllykangas' present address: Folkhälsan Institute of Genetics, University of Helsinki, Finland.

DOI:10.1523/JNEUROSCI.0285-07.2007

Copyright $\odot 2007$ Society for Neuroscience $\quad$ 0270-6474/07/273338-09\$15.00/0 central hydrophobic region of $\alpha$-synuclein, known as the non- $\beta$ amyloid component (NAC) of Alzheimer's disease senile plaques, is essential for aggregation of the protein. Deletion of residues 71-82 within the NAC region prevents $\alpha$-synuclein aggregation in vitro (Bodles et al., 2001; Giasson et al., 2001). A crucial role of NAC in $\alpha$-synuclein aggregation is also supported by the observation that the highly homologous $\beta$-synuclein, which differs from $\alpha$-synuclein in a 12 aa segment within the central part of the NAC domain, does not aggregate and is not found in Lewy inclusions (Biere et al., 2000). Conversely, the NAC region itself is highly amyloidogenic, readily forming fibrils that are similar to fibrils formed from other amyloidogenic proteins (Han et al., 1995; El-Agnaf et al., 1998a). Addition of NAC peptide aggregates is toxic to both PC12 and SHSY-5Y cells (ElAgnaf et al., 1998b; Bodles et al., 2001), although it is not clear that cells would be exposed to extracellular aggregates of $\alpha$-synuclein in vivo.

Similar in vitro studies have also suggested an inhibitory role for $\mathrm{C}$-terminal sequences of $\alpha$-synuclein on aggregation of the protein. Several investigators have shown that C-terminally truncated forms of recombinant $\alpha$-synuclein assemble into filaments more readily than the full-length wild-type (WT) protein (Crowther et al., 1998; Serpell et al., 2000; Murray et al., 2003). Some of these fragments are also efficient in seeding the aggregation of full-length $\alpha$-synuclein (Murray et al., 2003; Li et al., 2005; Liu et al., 2005). These findings may have relevance to Parkinson's disease because $\sim 15 \%$ of $\alpha$-synuclein found in Lewy inclusions is C-terminally truncated (Baba et al., 1998; Campbell et al., 2001; Giasson et al., 2002; Lee et al., 2002; Li et al., 2005; Liu et al., 2005). Thus, $\alpha$-synuclein may be proteolytically cleaved in vivo 
and such proteolysis may yield truncated species that accelerate $\alpha$-synuclein aggregation.

Although aggregation of $\alpha$-synuclein has been studied extensively in vitro, the influence of aggregation on $\alpha$-synuclein toxicity in vivo is less clear. In this study, we have used a Drosophila model of Parkinson's disease to investigate directly the role of $\alpha$-synuclein aggregation in mediating neurotoxicity. Transgenic flies expressing human $\alpha$-synuclein recapitulate essential features of the human disorder (Feany and Bender, 2000; Auluck et al., 2002). To assess the specific contribution of two major domains of $\alpha$-synuclein to toxicity and aggregation, we created transgenic lines expressing different mutant versions of human $\alpha$-synuclein: one containing a deletion in the NAC domain, and two C-terminally truncated variants.

\section{Materials and Methods}

Transgenic Drosophila. Drosophila were grown on standard cornmeal medium at $25^{\circ} \mathrm{C}$. The $\alpha-s y n^{\Delta 71-82}$ mutant was created as described by Giasson et al. (2001). The $\alpha$-syn ${ }^{1-120}$ and $\alpha-$ syn $^{1-87}$ constructs were generated by amplifying the wild-type $\alpha$-synuclein sequence using specific primers containing the start and stop codons. Constructs were cloned into the pUAST expression vector, and transgenic strains were created by embryo injection (Cutaneous Biology Research Center, Massachusetts General Hospital, Boston, MA). At least five independent transgenic lines were derived for each construct. The $D d c$-GAL4 driver we used drives expression in a substantial subset of dopaminergic neurons (Feany and Bender, 2000).

Immunocytochemical analysis. Adult flies were fixed in formalin and embedded in paraffin. Immunostaining on paraffin sections was performed using an avidin-biotin-peroxidase complex (Vectastain Elite; Vector Laboratories, Burlingame, CA) method as described previously (Feany and Bender, 2000). To examine dopaminergic neurons, flies were aged to $1,10,15$, and $20 \mathrm{~d}$, fixed, and immunostained for tyrosine hydroxylase (1:1000; ImmunoStar, Hudson, WI). Immunopositive cells were counted in well oriented frontal sections at the level of the giant fiber commissure, posterior to the fan-shaped body. At least 10 hemibrains were examined per genotype and time point. Quantitative experiments were performed with the investigator blinded to genotype to eliminate the possibility of investigator bias.

Quantitative analysis of the number of inclusions in cell cortex was determined in formic acid pretreated sections stained with the syn204 monoclonal antibody (1:1000; Zymed Laboratories, San Francisco, CA). The number of inclusions per $50 \mu \mathrm{m}^{2}$ length of well oriented brain was counted. At least eight hemibrains were examined per genotype.

Western blots. Adult fly heads of the appropriate genotypes were prepared and analyzed by standard SDS-PAGE using 10-20\% Tris-glycine gradient gels. The following $\alpha$-synuclein antibodies were used (see Fig. 6): clone 42, 1:20,000 (Transduction Laboratories, Lexington, KY); FL140, 1:20,000 (Santa Cruz Biotechnology, Santa Cruz, CA); syn204, 1:5000 (Zymed Laboratories); 7071-AP, 1:80,000 (affinity-purified, rabbit polyclonal antibody to recombinant, full-length human $\alpha$-synuclein); LB509, 1:1000, (Zymed Laboratories). An anti-actin from Sigma (St. Louis, MO) was used at 1:10,000.

Oligomer detection. To compare oligomer levels between $\alpha$-syn ${ }^{\mathrm{WT}}$ and $\alpha$-syn ${ }^{\Delta 71-82}$ transgenic flies, five heads per genotype and time point were homogenized at $4^{\circ} \mathrm{C}$ in TNE buffer (Lee et al., 2002) (10 mM Tris- $\mathrm{HCl}$ at pH7.5, $150 \mathrm{~mm} \mathrm{NaCl}, 5 \mathrm{~mm}$ EDTA) containing detergents (0.5\% Nonidet P-40) and protease inhibitors (Roche, Welwyn Garden City, UK). The homogenate was centrifuged at $16,000 \mathrm{~g}$ for $15 \mathrm{~min}$ and the resulting supernatant was analyzed by standard SDS-PAGE $10 \%$ Tris-glycine gel and immunoblot with a highly sensitive $\alpha$-synuclein antibody (7071-AP, $1: 20,000)$. Because the 7071-AP antibody does not recognize the monomeric form of $\alpha$-syn ${ }^{1-120}$ protein, different conditions were used to compare oligomer levels between $\alpha$-syn ${ }^{\text {WT }}$ and $\alpha$-syn ${ }^{1-120}$ transgenic flies. Two-hundred heads per genotype and time point were homogenized in TNE buffer and the homogenate was centrifuged at 100,000 $\mathrm{g}$ for $1 \mathrm{~h}$. The resulting supernatant was analyzed using clone 42 antibody (1:1000).

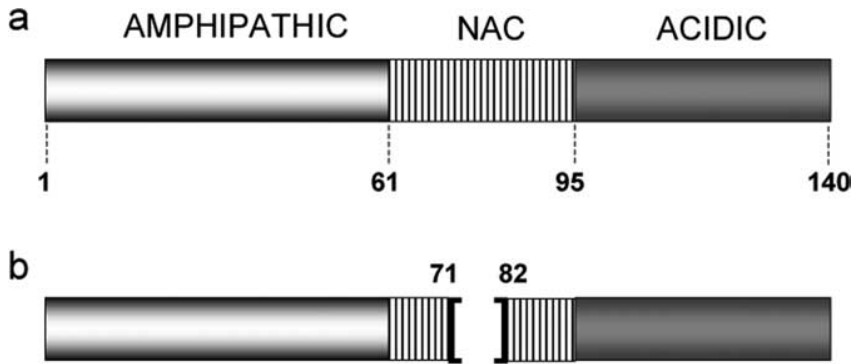

C

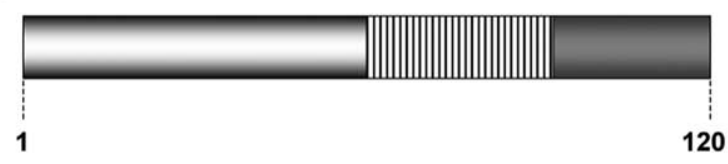

d

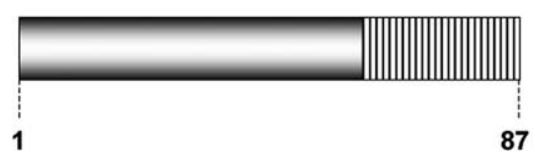

Figure 1. Schematic organization of the primary structure of $\alpha$-synuclein and three $\alpha$-synuclein mutants. $\boldsymbol{a}, \alpha$-Synuclein is a 140 aa protein that contains three major regions including an N-terminal amphipathic $\alpha$-helical domain (residues 1-67), a middle hydrophobic part (residues 61-95) known as the NAC of Alzheimer's disease senile plaques, and an acidic (-terminal region (residues 95-140). $\boldsymbol{a}$ - $\boldsymbol{d}$, Transgenic Drosophila expressing wild-type human $\alpha$-synuclein $(\boldsymbol{a}), \alpha$-synuclein with a deletion in the NAC domain from amino acids 71-82 (b), (-terminally truncated fragments comprising amino acids 1-120 (c), or amino acids $1-87$ (d) are analyzed.

Proteinase K digestion analysis. Paraffin-embedded tissue sections were deparaffinized with xylene and rehydrated to water. Digestion was performed with proteinase K at 0.7 (PK1) and $0.9 \mu \mathrm{g} / \mathrm{ml}^{-1}$ (PK2) in PBS at room temperature for $30 \mathrm{~min}$. Immunostaining was performed with clone 42 (1:200; Transduction Laboratories) and an Alexa Fluor 488conjugated goat anti-mouse secondary antibody (1:200; Invitrogen, Eugene, OR). Representative sections for each genotype and condition were acquired by confocal microscopy (Radiance laser-scanning confocal microscope; Bio-Rad, Hercules, CA). Before acquisition, laser parameters were adjusted to obtain nonsaturating conditions. All of the images for each figure were acquired in the same session using identical confocal acquisition parameters (laser power, gain, and pinhole settings).

\section{Results}

Based on the results of in vitro studies investigating $\alpha$-synuclein aggregation, we created transgenic Drosophila expressing several different forms of $\alpha$-synuclein: (1) a fragment with a deletion in the NAC domain that removes amino acids 71-82 $\left(\alpha-\right.$ syn $\left.^{\Delta 71-82}\right)$, (2) a C-terminally truncated fragment comprising amino acids $1-120\left(\alpha\right.$-syn $\left.{ }^{1-120}\right)$, and (3) a C-terminally truncated fragment comprising amino acids $1-87\left(\alpha\right.$-syn $\left.{ }^{1-87}\right)$ (Fig. 1$)$. Complementary DNA constructs were cloned under the control of upstream activating sequence (UAS) for the yeast transcription factor GAL4, and we used the GAL4-UAS bipartite system to direct $\alpha$-synuclein expression to the brain using the pan-neuronal driver elav-GAL4. Quantitative Western blot analyses were performed on fly heads to select lines expressing the mutant form of $\alpha$-synuclein at levels equivalent to that of the control wild-type $\alpha$-synuclein transgenic line. Lines with equivalent levels of expression were recovered for $\alpha$-syn ${ }^{\Delta 71-82}$ and $\alpha$-syn ${ }^{1-120}$ (supplemental Fig. S1, available at www.jneurosci.org as supplemental material). However, all of the transgenic lines expressing 
$\alpha$-syn ${ }^{1-87}$ had significantly lower levels of expression than flies expressing wild-type $\alpha$-synuclein. The highest expressing line of $\alpha$-syn ${ }^{1-87}$ that we recovered had approximately sixfold less $\alpha$-synuclein than our control wild-type $\alpha$-synuclein transgenic animals. We also attempted to express the NAC domain alone (amino acids 61-95); however, we did not recover animals expressing detectable levels of this short protein.

\section{The NAC domain is essential for $\alpha$-synuclein toxicity to dopaminergic neurons}

To assess the impact of $\alpha$-syn ${ }^{\Delta 71-82}$ on dopaminergic neurons, we immunostained brains from flies at 1 and $20 \mathrm{~d}$ posteclosion with an antibody against tyrosine hydroxylase, which specifically identifies these neurons. We analyzed a dorsomedial group of dopaminergic neurons because these neurons are easily identifiable and preferentially sensitive to $\alpha$-synuclein toxicity (Feany and Bender, 2000; Auluck et al., 2002; Yang et al., 2003). We used a highly sensitive immunoperoxidase detection method (see Materials and Methods) to ensure optimal visualization of dopaminergic neurons and tissue sections with well defined anatomic landmarks to delineate the vulnerable dorsomedial neurons. Under these conditions, we interpret the loss of tyrosine hydroxylase immunostaining we observe as loss of dopaminergic neurons. In contrast, other groups have suggested that apparently normal numbers of dopaminergic neurons reported in $\alpha$-synuclein transgenic flies analyzed with whole-mount immunostaining techniques reflect dysfunction rather than death of these neurons (Auluck et al., 2005; Pesah et al., 2005).

To address the issue of cell death versus dysfunction, we expressed the anti-apoptotic proteins thread (Hay et al., 1995) and p35 (Clem et al., 1991) in dopaminergic neurons and protected from $\alpha$-synuclein-mediated neuronal loss (supplemental Fig. S2, available at www.jneurosci.org as supplemental material). Because thread and p35 have well characterized roles in blocking apoptosis, these findings are consistent with our view that dopaminergic cell death is occurring in our model. We cannot, however, completely exclude the possibility that overexpression of these antiapoptotic proteins improves the overall health of dopamine neurons rather than rescuing cell death. Additional work will be needed to address definitively the role of cell death versus dysfunction in our model.

In nontransgenic flies, the dorsomedial neurons we study are represented throughout life by four or five cells showing robust staining for tyrosine hydroxylase-cells. Similarly, in young adult flies expressing $\alpha$-syn ${ }^{\mathrm{WT}}$ or $\alpha$-syn ${ }^{\Delta 71-82}$, the dorsomedial cluster consisted of the normal number of dopaminergic neurons (Fig. $2 a, b)$. At day 20 however, adult flies expressing $\alpha$-syn ${ }^{\text {WT }}$ demonstrated a marked loss of tyrosine hydroxylase-positive cells in this cluster (Fig. $2 c, e$ ), as shown previously in our model (Feany and Bender, 2000). In contrast, aged transgenic flies expressing $\alpha$-syn ${ }^{\Delta 71-82}$ showed no loss of tyrosine hydroxylase staining in these dopaminergic neurons (Fig. 2d,e). Thus, amino acids 71-82 in the NAC domain of $\alpha$-synuclein are essential for toxicity to dopaminergic neurons.

\section{The NAC domain is required for $\boldsymbol{\alpha}$-synuclein aggregation}

Flies expressing wild-type $\alpha$-synuclein show progressive accumulation of cytoplasmic aggregates resembling Lewy bodies and Lewy neurites (Feany and Bender, 2000). To determine whether a deletion in the NAC domain affects $\alpha$-synuclein aggregation in vivo, we monitored inclusion formation in the brains of flies expressing $\alpha$-syn ${ }^{\Delta 71-82}$ by performing $\alpha$-synuclein immunostaining on brain tissue sections. Although neuronal inclusions were a

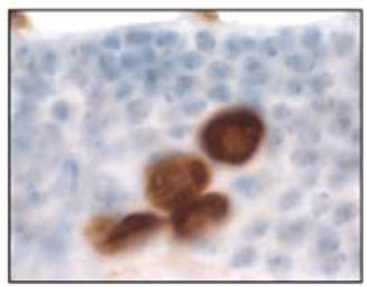

C

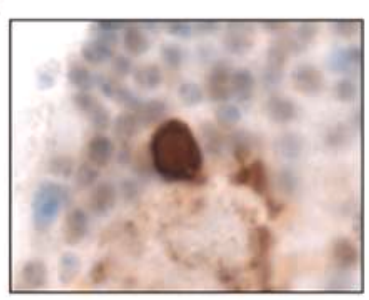

b

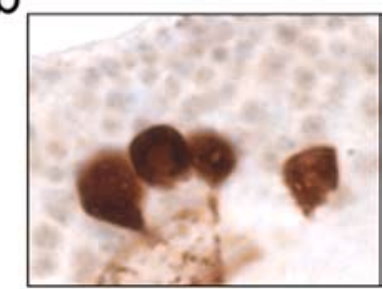

d

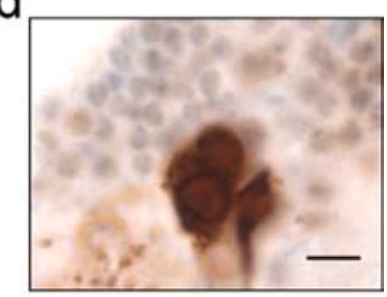

\section{e}

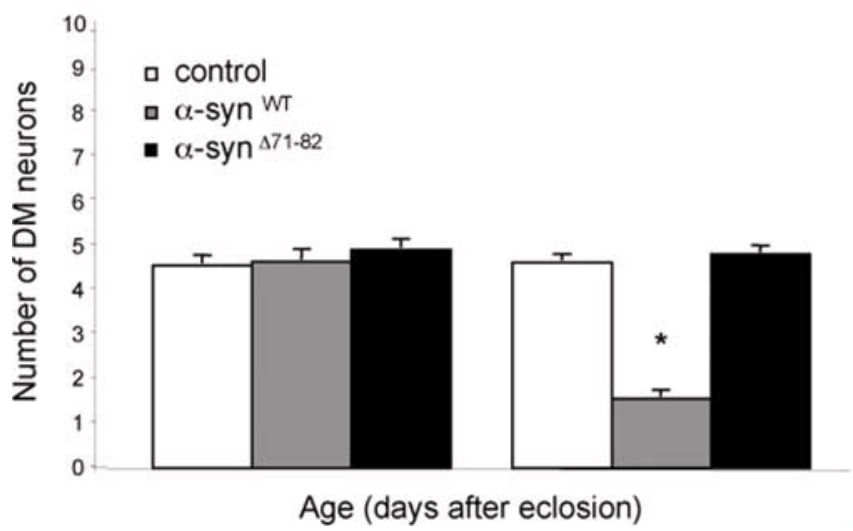

Figure 2. The NAC domain is essential for $\alpha$-synuclein toxicity to dopaminergic neurons. $\boldsymbol{a}$, $\boldsymbol{b}$, Tyrosine hydroxylase immunostaining identifies a normal number of dorsomedial neurons in 1-d-old flies expressing wild-type $\alpha$-synuclein ( $\boldsymbol{a}$ ) or $\alpha$-syn ${ }^{\Delta 71-82}(\boldsymbol{b})$ in a pan-neural pattern with the elav-GAL4 driver. c, Loss of tyrosine hydroxylase-immunoreactivity is observed in 20$\mathrm{d}$-old $\alpha$-syn ${ }^{\mathrm{WT}}$ transgenic flies. $\boldsymbol{d}$, Neurons are preserved in 20-d-old $\alpha$-syn ${ }^{\Delta 71-82}$ transgenic flies. Scale bar: (in $\boldsymbol{d}) \boldsymbol{a}-\boldsymbol{d}, 5 \mu \mathrm{m}$. $\boldsymbol{e}$, Quantitative analysis of dopaminergic neuron numbers. Values represent mean $\pm S E M$. Asterisk indicates that the difference in dopaminergic neuron number between 20-d-old $\alpha$-syn ${ }^{\Delta 71-82}$ and $\alpha$-syn ${ }^{\Delta 71-82}$ flies is statistically significant ( $p<$ 0.01 , multivariate ANOVA with supplementary Newman-Keuls test). No significant difference is present in cell numbers between $\alpha$-syn ${ }^{\Delta 71-82}$ flies and elav-GAL 4 heterozygous control flies at $20 \mathrm{~d}$.

observed in the cell cortex and neuropil of 20-d-old flies expressing wild-type $\alpha$-synuclein (Fig. $3 a, c$ ), no inclusions were observed in flies expressing $\alpha$-syn ${ }^{\Delta 71-82}$ at any age (Fig. $3 b, d$ ).

$\alpha$-Synuclein appears to form a variety of smaller species, often termed oligomers or protofibrils, before their aggregation into filaments. There is extensive speculation that these smaller aggregates may be the most relevant neurotoxic species in vivo (Volles and Lansbury, 2003; Cookson, 2005). We thus investigated whether flies expressing the $\alpha$-syn ${ }^{\Delta 71-82}$ mutant still have the ability to form oligomeric species. To detect soluble oligomeric forms of $\alpha$-synuclein, we analyzed soluble fractions of fly brain homogenates by Western blotting, using a sensitive $\alpha$-synuclein antibody (7071-AP). We identified several higher molecular weight forms of $\alpha$-synuclein in 20-d-old wild-type $\alpha$-synuclein transgenic flies, with a major species running at $190 \mathrm{kDa}$ (Fig. $3 e$ ). These oligomeric forms were not present in 1-d-old flies but 
Figure 3. The NAC domain is essential for $\alpha$-synuclein aggregation. $\boldsymbol{a}, \boldsymbol{c}, \alpha$-Synuclein staining identified inclusions (arrows) in the lamina cell cortex (a) and in the neuropil (c) of $20 \mathrm{~d} \alpha$-syn ${ }^{\text {WT }}$ flies. $\boldsymbol{b}, \boldsymbol{d}$, In contrast, no inclusions are observed in $\alpha$-syn ${ }^{\Delta 71-82}$ flies of the same age. Scale bar: (in $\boldsymbol{d}) \boldsymbol{a}-\boldsymbol{d}, 5 \mu \mathrm{m} . \boldsymbol{e}$, Western blot analysis with the 7071-AP antibody of soluble fractions from brain homogenates run on a $10 \%$ gel identifies several high molecular weight forms of $\alpha$-synuclein in 20 - $d$-old $\alpha$-syn ${ }^{\text {WT }}$ flies only, with a major species running at $190 \mathrm{kDa}$. None of these oligomeric species are present in $\alpha$-syn ${ }^{\Delta 71-82}$ flies or in nontransgenic elav-GAL4/+ control flies. Aliquots of the same protein preparations are loaded on a $10-20 \%$ gel to visualize the monomeric forms of $\alpha$-synuclein. Equivalent protein loading of protein is shown by stripping the membrane and reprobing it with an anti-actin antibody. $\boldsymbol{f}$, Flies expressing wild-type $\alpha$-synuclein in dopaminergic neurons using the Ddc-GAL4 driver have no detectable proteinase K-resistant $\alpha$-synuclein at 10 d. Proteinase K treatment of brains from 20 - $d$-old flies show proteinase K-resistant $\alpha$-synuclein in wild-type expressing flies only.

started to appear in 10-d-old flies (visible on longer exposure) (data not shown), showing a progressive accumulation of these oligomers with age. None of these soluble oligomeric species were observed in $\alpha$-syn ${ }^{\Delta 71-82}$ transgenic flies, or in nontransgenic controls. Equivalent levels of the monomeric form of $\alpha$-synuclein were detected in all of the genotypes at the different time points (Fig. 3e). Thus, flies expressing $\alpha$-syn ${ }^{\Delta 71-82}$ are unable to form small or large aggregates of $\alpha$-synuclein.

Resistance to proteinase $\mathrm{K}$ digestion is a common feature of misfolded $\alpha$-synuclein in Parkinson's disease and other neurodegenerative disorders (Neumann et al., 2002). We expressed wild- type $\alpha$-synuclein or $\alpha$-syn ${ }^{\Delta 71-82}$ in dopaminergic neurons using the Ddc-GAL4 driver line that drives expression specifically in dopaminergic neurons (Feany and Bender, 2000; Li et al., 2000) (supplemental Fig. S3, available at www.jneurosci.org as supplemental material). These flies had no detectable proteinase K-resistant $\alpha$-synuclein at day 10 (Fig. $3 f$ ). However, 20-d-old flies expressing wild-type $\alpha$-synuclein developed proteinase $\mathrm{K}$ resistance, which was not observed in $\alpha$-syn ${ }^{\Delta 71-82}$ transgenic flies (Fig. 3f). Proteinase $\mathrm{K}$-resistant $\alpha$-synuclein was punctate in appearance (supplemental Fig. S4, available at www.jneurosci.org as supplemental material), consistent with aggregate formation.

\section{The C-terminal domain influences $\alpha$ -} synuclein toxicity and aggregation Because C-terminally truncated forms of $\alpha$-synuclein are present in vivo and aggregate more readily than full-length $\alpha$-synuclein in vitro, we assessed whether expressing truncated fragments of $\alpha$-synuclein in flies could affect neurotoxicity (Fig. 4). Quantitative analysis of dopaminergic neurons showed that 1-d-old transgenic flies expressing $\alpha$-syn ${ }^{1-120}$ had a normal number of dopaminergic neurons in the dorsomedial cluster. Thus, expression of a C-terminally truncated form of $\alpha$-synuclein, like expression of wildtype $\alpha$-synuclein, did not disrupt the development of dopaminergic neurons. However, there was a modest, but statistically significant increase in neurotoxicity as seen by decreased numbers of tyrosine hydroxylase-positive dorsomedial neurons at $15 \mathrm{~d}$ in $\alpha$-syn ${ }^{1-120}$ transgenic flies compared with flies expressing wild-type $\alpha$-synuclein. By day 20, the marked loss of tyrosine hydroxylase immunostaining in cells in this cluster was similar between wild-type $\alpha$-synuclein transgenics and $\alpha$-syn ${ }^{1-120}$ transgenic flies. Thus, C-terminal truncation of $\alpha$-synuclein after amino acid 120 modestly enhances the toxicity of the protein. We also examined flies expressing $\alpha$-syn ${ }^{1-120}$ along with wild-type $\alpha$-synuclein. No dramatic enhancement of neurotoxicity was observed in these animals compared with flies expressing two copies of wild-type $\alpha$-synuclein (data not shown).

We also examined the toxicity of $\alpha$-synuclein truncated after residue 87, but saw no dopaminergic neurotoxicity in $\alpha$-syn ${ }^{1-87}$ transgenic flies (data not shown). Because we were unable to express levels of $\alpha$-syn ${ }^{1-87}$ equivalent to the other $\alpha$-synuclein variants we studied, we cannot definitively compare the toxicity of this truncated fragment compared with full-length $\alpha$-synuclein or $\alpha$-syn ${ }^{1-120}$, but our results do suggest that $\alpha$-syn ${ }^{1-87}$ is not highly toxic at lower levels of expression. We also 


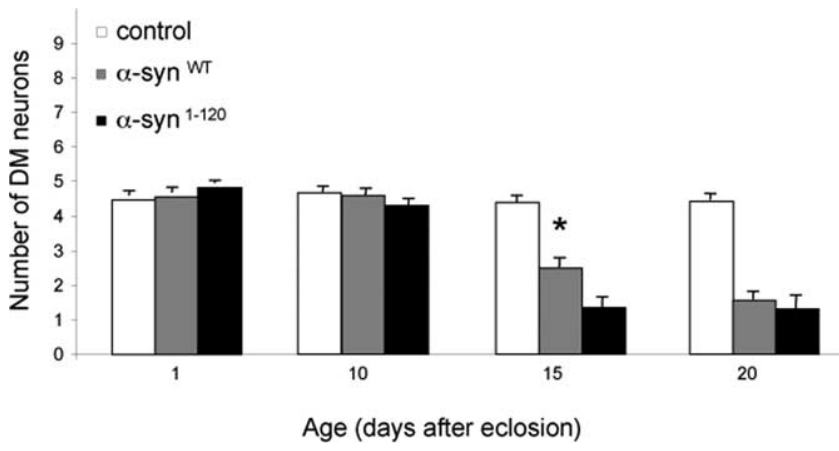

Figure 4. The C-terminal domain represses $\alpha$-synuclein toxicity. Quantitative analysis of tyrosine hydroxylase-positive dopaminergic neuron numbers among flies expressing wild-type $\alpha$-synuclein and $\alpha$-syn ${ }^{1-120}$ and elav-GAL4/+ controls. Values represent mean \pm SEM. Asterisk indicates that the difference in dopaminergic neuron number between wild-type $\alpha$-synuclein and $\alpha$-syn ${ }^{1-120}$ transgenic flies is statistically significant at day $15(p<0.05)$.

created flies expressing $\alpha$-syn ${ }^{1-87}$ along with wild-type $\alpha$-synuclein. No dramatic enhancement of neurotoxicity or inclusion formation was evident in these animals.

To determine whether enhanced neurotoxicity of $\alpha$-syn ${ }^{1-120}$ correlated with increased insoluble $\alpha$-synuclein, we immunostained sections of brains to identify inclusion bodies. Compared with flies expressing wild-type $\alpha$-synuclein (Fig. 5a), there were significantly increased numbers of cytoplasmic $\alpha$-synuclein immunoreactive inclusions in aged flies expressing $\alpha$-syn ${ }^{1-120}$ (Fig. 5b,c).

We also compared the resistance to proteinase $\mathrm{K}$ digestion of wild-type $\alpha$-synuclein or $\alpha$-syn ${ }^{1-120}$ in dopaminergic neurons (Fig. $5 d$ ). No detectable proteinase K resistance was observed in 1-d-old flies (Chen and Feany 2005) (data not shown). In contrast, treatment of sections from aged fly brains with increasing concentrations of proteinase $\mathrm{K}$ revealed increased proteinase $\mathrm{K}$ resistance in $\alpha$-syn ${ }^{1-120}$ transgenics flies compared with wildtype flies of the same age. At day 10, the majority of wild-type $\alpha$-synuclein was proteinase $\mathrm{K}$ sensitive whereas $\alpha$-syn ${ }^{1-120}$ showed resistance to proteinase $\mathrm{K}$ (Fig. $5 d$ ). As the transgenic flies aged, both wild-type $\alpha$-synuclein and $\alpha$-syn ${ }^{1-120}$ flies showed clear resistance to proteinase $\mathrm{K}$ (Fig. $5 d$, PK1). However, at a higher proteinase K concentration, only $\alpha$-syn ${ }^{1-120}$ flies showed resistance to proteinase $\mathrm{K}$ (Fig. $5 d$, PK2).

We also examined aggregated forms of $\alpha$-synuclein by Western blot analysis in our transgenic flies expressing truncated forms of $\alpha$-synuclein. The sensitive antibody used in Figure $3 e$ does not recognize the monomeric forms of $\alpha$-syn ${ }^{1-120}$ or $\alpha$-syn ${ }^{1-87}$ variants under denaturing SDS-PAGE conditions, and we were unable to detect reliably the $\sim 190 \mathrm{kDa}$ species using less sensitive antibodies in $\alpha$-syn ${ }^{1-120}$ transgenic flies. However, higher molecular weight species were easily detected in $\alpha$-syn ${ }^{1-}$ 120 transgenic flies (Fig. 5e). These aggregates appeared earlier in $\alpha$-syn ${ }^{1-120}$ transgenic flies and accumulated at higher levels in flies expressing the truncation compared with flies expressing wild-type $\alpha$-synuclein (Fig. 5e,f).

\section{$\alpha$-synuclein is naturally truncated in Drosophila}

Because truncated forms of $\alpha$-synuclein have been identified in human brains as well as in $\alpha$-synuclein transgenic mice, we wondered whether these fragments were also present in $\alpha$-synuclein transgenic flies. Western blot analysis of 1-d-old flies expressing wild-type $\alpha$-synuclein identified two bands: a major product migrating $\sim 14 \mathrm{kDa}$ corresponding to the full-length form of the protein, and a minor product migrating $\sim 12 \mathrm{kDa}$ (Fig. $6 a$ ). In- terestingly, this low-molecular weight form of the protein showed the same electrophoretic mobility as $\alpha$-syn ${ }^{1-120}$. Similar amounts of the lower-molecular weight species were formed in $\alpha$-syn ${ }^{\Delta 71-82}$ transgenic flies. We further characterized this lowmolecular weight form of $\alpha$-synuclein using a panel of antibodies recognizing epitopes along the length of the protein (Fig. 6b). Only $\alpha$-synuclein antibodies with epitopes localized N-terminal to amino acid 120 of the protein recognized the low-molecular weight form of $\alpha$-synuclein. Thus, $\alpha$-synuclein undergoes physiological truncation in transgenic Drosophila, and this cleavage is not altered by removing residues 71-82 within the NAC domain of the protein.

\section{Discussion}

A remarkable feature of virtually all human neurodegenerative diseases is the presence of insoluble protein aggregates in brains of patients with the disorders. These aggregates may involve the neuronal cell body or process, as in Lewy inclusions, or may form extracellularly or even in glial cells. The presence of these inclusion bodies has led many investigators to implicate abnormalities in protein aggregation as a key feature underlying the pathogenesis of many neurodegenerative diseases, including Parkinson's disease (Trojanowski and Lee, 2000; Cookson, 2005). In the case of Parkinson's disease, the synaptic protein $\alpha$-synuclein both aggregates in Lewy bodies and Lewy neurites and is genetically implicated in familial forms of the disorder. Thus, significant effort has been expended in defining the regions of $\alpha$-synuclein that control aggregation of the protein in vitro. We have used the information provided by these in vitro experiments to test the importance of $\alpha$-synuclein aggregation in vivo in our Drosophila model that recapitulates many features of Parkinson's disease, including selective dopaminergic neurotoxicity. Our data provide strong evidence supporting the role of $\alpha$-synuclein aggregates in mediating neurotoxicity. A deletion mutation in $\alpha$-synuclein that abolishes aggregation in vitro also eliminates soluble oligomers and large inclusion body formation in vivo and prevents neurotoxicity, whereas a C-terminal truncation that promotes aggregation in vitro increases the formation of several aggregated species in vivo and enhances neurotoxicity.

A number of lines of evidence suggest that the insoluble aggregates classically identified by light microscopy may not themselves comprise neurotoxic species. In the case of neurodegeneration caused by abnormal expansion of polyglutamine encoding trinucleotide repeats, several studies have suggested that large aggregates may play a protective, rather than pathological role (Saudou et al., 1998; Arrasate et al., 2004). In $\alpha$-synuclein transgenic Drosophila, expression of human Hsp70 or pharmacological upregulation of heat-shock proteins rescues dopaminergic toxicity, but does not decrease inclusion formation (Auluck et al., 2002, 2005). Our own laboratory has presented evidence that preventing phosphorylation at Ser129 of $\alpha$-synuclein prevents neurotoxicity, while increasing the numbers of large inclusion bodies in transgenic flies (Chen and Feany, 2005).

How can a protective role for inclusions be reconciled with the data presented here that strongly implicate aggregation of $\alpha$-synuclein in neurotoxicity? A number of in vitro studies suggest the following model of $\alpha$-synuclein aggregation: starting with the natively unfolded monomers, the system populates a heterogeneous mixture of protofibrils, at least some of which are consumed as fibrils form. Fibrils of $\alpha$-synuclein then coalesce to form the Lewy bodies seen by light microscopy in the brains of patients with Parkinson's disease (Cookson, 2005). $\alpha$-Synuclein protofibrils rather than the fibrils themselves may be the patho- 
a

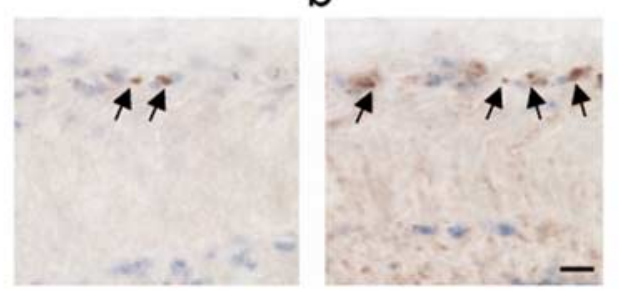

C
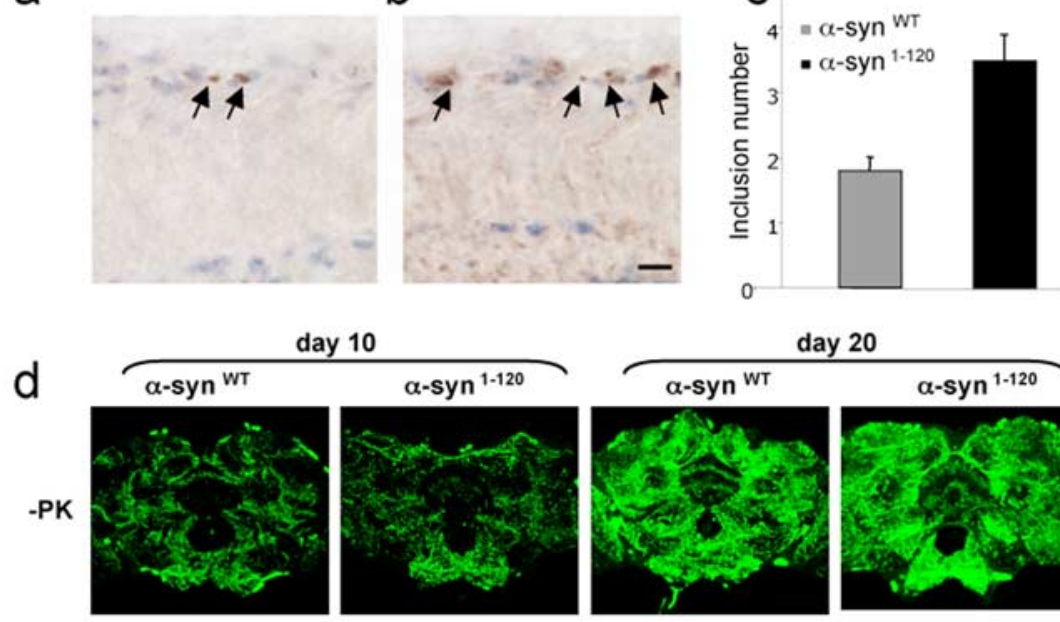

day 10
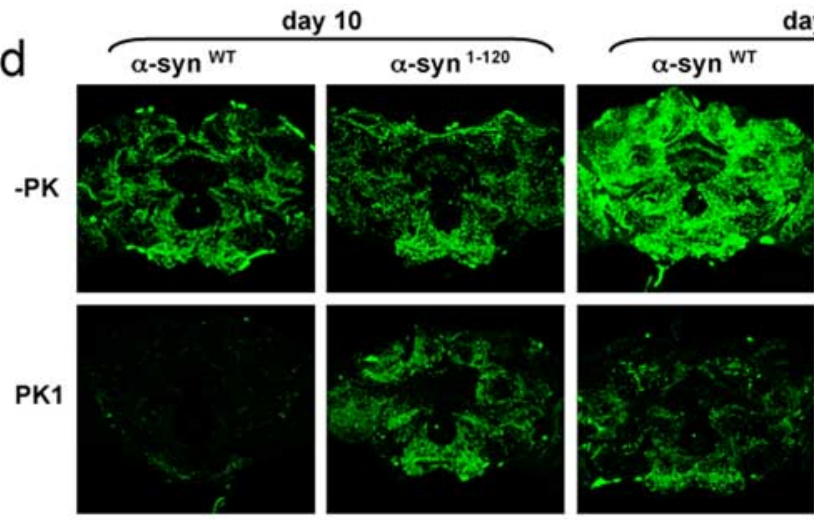

day 20
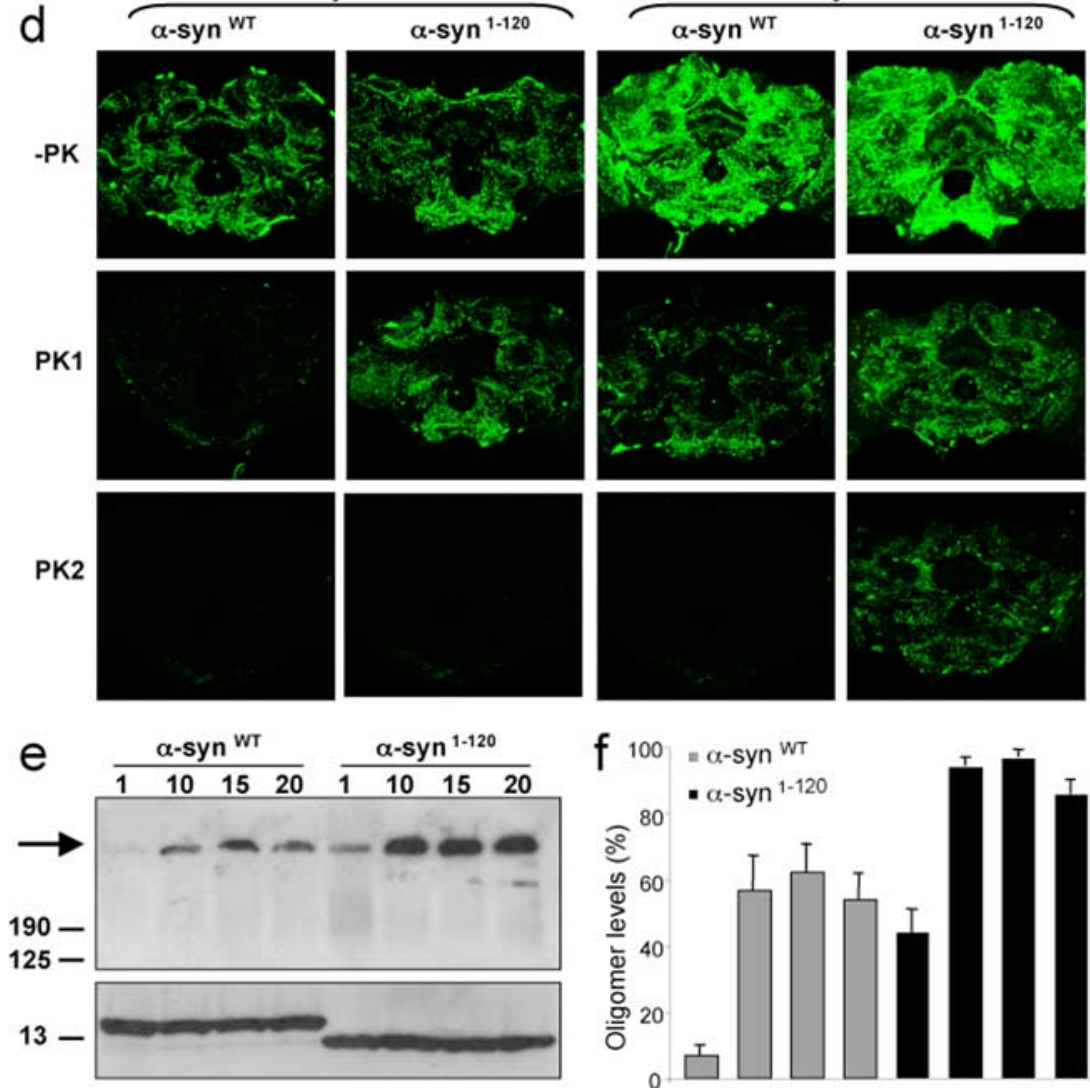

f $100=\alpha$-syn $w T$

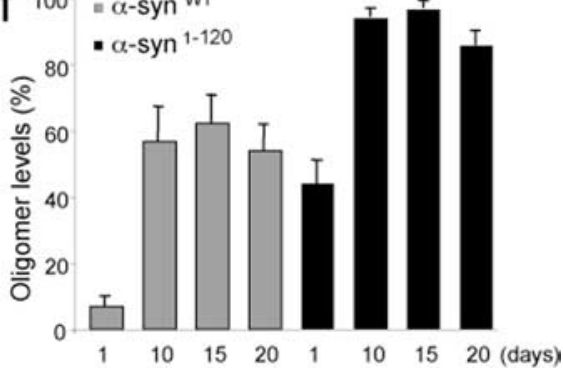

Figure 5. The C-terminal domain represses $\alpha$-synuclein aggregation. $\boldsymbol{a}, \boldsymbol{b}, \alpha$-Synuclein immunostaining identifies moderate numbers of inclusions in the lamina cell cortex in flies expressing wild-type $\alpha$-synuclein $(\boldsymbol{a})$ and increased numbers of inclusions (arrows) in the cortex of flies expressing $\alpha$-syn ${ }^{1-120}$ at $20 \mathrm{~d}(\boldsymbol{b})$. Scale bar, $5 \mu \mathrm{m}$. c, Quantitative analysis of inclusions numbers. Values represent mean \pm SEM. The difference is statistically significant $(p<0.01$, Student's $t$ test). $\boldsymbol{d}$, Increased inclusion formation correlates with increased proteinase $K$ resistance. Proteinase $K$ treatment of brains from 10 - and 20-d-old flies show increased proteinase K-resistant $\alpha$-synuclein in flies expressing $\alpha$-syn ${ }^{1-120}$ compared with flies expressing wild-type $\alpha$-synuclein. At $10 \mathrm{~d}$, flies expressing wild-type $\alpha$-synuclein in dopaminergic neurons have no detectable proteinase K-resistant $\alpha$-synuclein, whereas resistant $\alpha$-synuclein is observed in $\alpha$-syn ${ }^{1-120}$. At $20 \mathrm{~d}$, both wild-type $\alpha$-synuclein and $\alpha$-syn ${ }^{1-120}$ flies demonstrate resistance to proteinase K at a low concentration of proteinase K (PK1), but only $\alpha$-syn ${ }^{1-120}$ show resistance at a higher proteinase K concentration (PK2). $\boldsymbol{e}$, Western blot analysis with the Clone42 antibody of soluble fractions from brain homogenates run on a $10 \%$ gel identified high molecular weight forms of $\alpha$-synuclein (arrow) with an earlier and increased accumulation in $\alpha$-syn ${ }^{1-120}$ compared with $\alpha$-syn ${ }^{\text {WT }}$. Aliquots of the same protein preparations are loaded on a $10-20 \%$ gel to visualize the monomeric forms of $\alpha$-synuclein. $f$, Intensities of the corresponding bands are quantitated by densitometry using NIH ImageJ. Readings from $\alpha$-syn ${ }^{1-120}$ are normalized to $100 \%$ and relative intensity ratios are calculated. Values represent mean \pm SEM. ANOVA test with a Student-Newman-Keuls post-test shows significant differences between the two genotypes for each time point examined.

genic species. Although all of the Parkinson's disease-linked mutations enhance the aggregation of $\alpha$-synuclein (Conway et al., 1998; Giasson et al., 1999; Narhi et al., 1999; Greenbaum et al., 2005), the A30P mutation increases the amount of oligomeric species at the expense of fibrils (Conway et al., 2000; J. Li et al., 2002). Our observation of soluble oligomeric forms in our $\alpha$-synuclein transgenic flies (Fig. 3 ) is consistent with the notion of small toxic aggregates as biologically relevant intermediates.

Further supporting an important role for protein aggregation in our model, C-terminal truncation of $\alpha$-synuclein increases neurotoxicity while increasing the numbers of inclusion bodies, enhancing proteinase $\mathrm{K}$ resistance and increasing the levels of high molecular weight species of $\alpha$-synuclein on Western blots of $\alpha$-syn ${ }^{1-120}$ transgenic flies (Figs. 4, 5). Because all of these species of aggregated $\alpha$-synuclein track with toxicity, the present study does not allow us to distinguish which of these species are relevant for toxicity. We feel that large inclusions are unlikely to have substantial toxicity because we have demonstrated previously increased inclusions in the context of neuroprotection (Chen and Feany, 2005). We were not able to analyze all oligomeric species present in wild-type $\alpha$-synuclein transgenic flies in $\alpha$-syn ${ }^{1-120}$ flies, so we are limited in our ability to implicate a particular oligomeric species in toxicity.

C-terminal truncation is likely to be physiologically relevant because a fragment of $\alpha$-synuclein similar to the $\alpha$-syn ${ }^{1-120}$ we expressed in our transgenic flies is seen in wild-type $\alpha$-synuclein transgenic flies (Fig. 6), in patients with Parkinson's disease, and related $\alpha$-synucleinopathies (Baba et al., 1998; Campbell et al., 2001) and in transgenic mice expressing full-length $\alpha$-synuclein (Giasson et al., 2002; Lee et al., 2002; Li et al., 2005; Liu et al., 2005). C-terminally truncated forms of $\alpha$-synuclein are present in Lewy inclusions in patients (Baba et al., 1998), and are selectively recovered in insoluble fractions from synucleinopathy patients (Tofaris et al., 2003; Li et al., 2005) and $\alpha$-synuclein transgenic mice ( $\mathrm{Li}$ et al., 2005). These findings correlate well with in vitro studies demonstrating that recombinant truncated $\alpha$-synuclein has an enhanced propensity to aggregate (Crowther et al., 1998; Serpell et al., 2000; H. T. Li et al., 2002; Murray et al., 2003).

Although our findings of enhanced neurotoxicity and increased aggregation in $\alpha$-syn ${ }^{1-120}$ are generally consistent with previous in vivo and in vitro work on C-terminal truncations of $\alpha$-synuclein, the enhanced neurotoxicity we observe in flies expressing $\alpha$-syn ${ }^{1-120}$ is modest overall (Fig. 4). These findings are in accordance with a recent study investigating the effects of $\alpha$-synuclein C-terminal truncation by generating mice expressing human $\alpha$-syn ${ }^{1-120}$ from the rat tyrosine hydroxylase promoter (Tofaris et al., 2006). Although mixed granular and fibrillar aggregates 

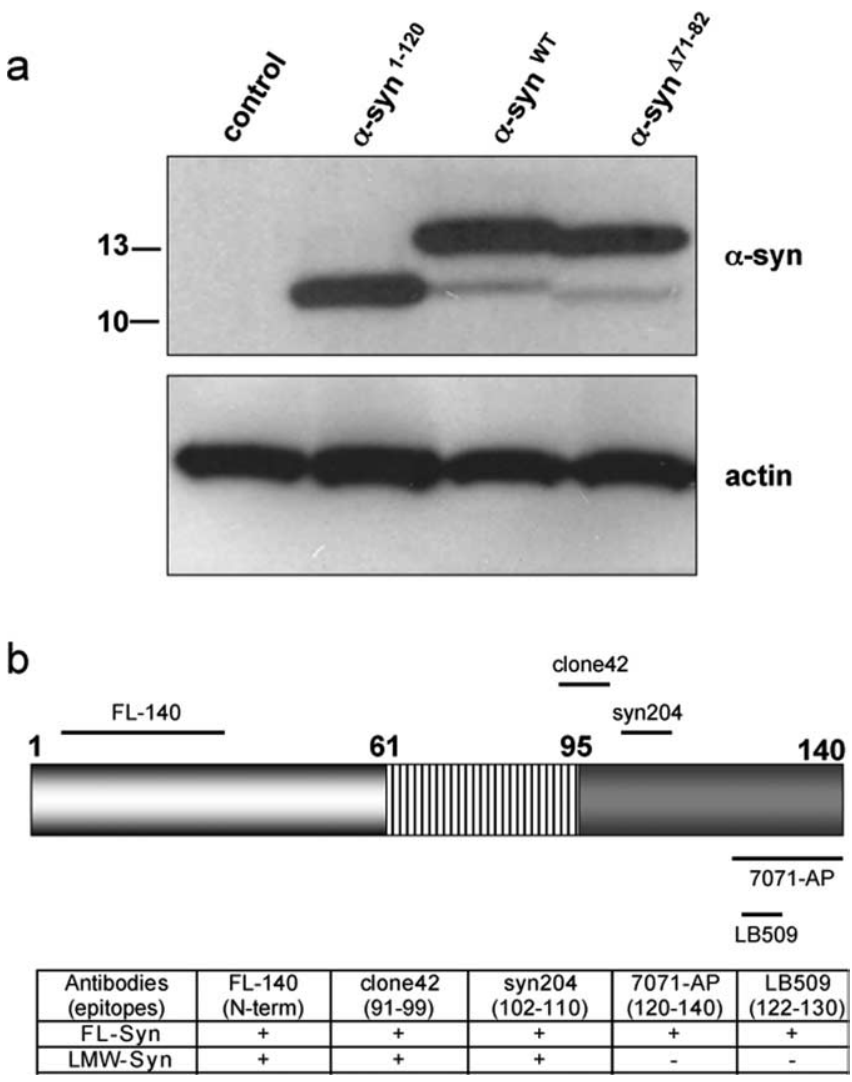

Figure 6. $\quad \alpha$-Synuclein is naturally truncated in Drosophila. a, SDS-PAGE analysis of 1-d-old flies expressing $\alpha$-synuclein identified two bands: a major product migrating at $14 \mathrm{kDa}$ and corresponding to the full-length form of the protein, and a minor product migrating at $12 \mathrm{kDa}$ at the same position as $\alpha$-syn ${ }^{1-120}$. This low molecular weight form of $\alpha$-synuclein is also present in flies expressing $\alpha$-syn ${ }^{\Delta 71-82}$. $\boldsymbol{b}$, Characterization of the low molecular weight form of $\alpha$-synuclein. Only $\alpha$-synuclein antibodies with epitopes localized before amino acid 120 recognize the low molecular weight form of $\alpha$-synuclein. FL, Full length; LMW, low molecular weight.

were detected in olfactory bulb and substantia nigra of 12month-old mice, no dopaminergic cell loss was observed. Transgenic mice expressing another C-terminally truncated form of $\alpha$-synuclein $\left(\alpha\right.$-syn $\left.{ }^{1-130}\right)$ and carrying the A53T point mutation show a nonprogressive loss of dopaminergic neurons without evidence of Lewy bodies inclusions (Wakamatsu et al., 2007).

The inability of truncated forms of $\alpha$-synuclein to produce substantial, progressive neurotoxicity in vivo could reflect a more moderate effect of the chosen C-terminal truncation on neurotoxicity in vivo compared with aggregation potential in vitro. Alternatively, substoichiometric levels of truncated $\alpha$-synuclein in vivo may primarily act as a seed for aggregation of full-length $\alpha$-synuclein (Li et al., 2005; Liu et al., 2005). Because we did not recover transgenic lines expressing markedly lower levels of $\alpha$-syn ${ }^{1-120}$ compared with wild-type $\alpha$-synuclein, we were unable to test this hypothesis rigorously in our system. We did analyze flies expressing equivalent levels of $\alpha$-syn ${ }^{1-120}$ and wild-type $\alpha$-synuclein, but did not observe significantly increased neurotoxicity or aggregation in these animals that would suggest a synergistic rather than simply additive effect.

We also addressed potential seeding of wild-type $\alpha$-synuclein aggregation by our low-expressing $\alpha$-syn ${ }^{1-87}$ line, but did not observe dramatic enhancement of either neurotoxicity or inclusion formation when we expressed wild-type $\alpha$-synuclein along with sixfold lower levels of $\alpha$-syn ${ }^{1-87}$. Observing a seeding effect of truncated $\alpha$-synuclein variants may require more precise titration of mutant versus wild-type protein. Alternatively, truncated fragments of $\alpha$-synuclein may play a less prominent role in aggregation and neurotoxicity in vivo than suggested by in vitro studies.

A potential role for C-terminally truncated fragments in seeding aggregation of wild-type $\alpha$-synuclein is also intriguing because a number of post-translational modifications of $\alpha$-synuclein, including phosphorylation (Fujiwara et al., 2002; Chen and Feany, 2005) or nitration (Giasson et al., 2000; Yamin et al., 2003), or methionine oxidation (Glaser et al., 2005; Mirzaei et al., 2006), which appear to play an important role in controlling $\alpha$-synuclein aggregation and toxicity, selectively target the last 20 aa of $\alpha$-synuclein. These important modifications, thus, cannot affect toxicity in transgenic flies expressing $\alpha$-syn ${ }^{1-120}$ in the absence of longer forms of $\alpha$-synuclein.

Although C-terminally truncated $\alpha$-synuclein might play a more significant role in modulating neurotoxicity when present at low levels together with full-length $\alpha$-synuclein, our data suggest that any such toxic role for truncated $\alpha$-synuclein depends on protein aggregation. $\alpha$-Synuclein lacking residues $71-82(\alpha-$ $\operatorname{syn}^{\Delta 71-82}$ ) is still efficiently truncated at its $C$ terminus (Fig. 6). However, transgenic animals expressing $\alpha$-syn ${ }^{\Delta 71-82}$ show no evidence of dopaminergic dysfunction. Thus, an intact NAC domain is not required for efficient truncation of $\alpha$-synuclein in vivo, but NAC function, presumably to mediate aggregation, is still needed for neurotoxicity even in the presence of C-terminally truncated fragments.

In conclusion, we provide strong evidence that aggregated forms of $\alpha$-synuclein mediate dopaminergic neurotoxicity in a Drosophila model of Parkinson's disease. In our in vivo system, the NAC domain of $\alpha$-synuclein is absolutely required for aggregation and dopaminergic neurotoxicity whereas sequences C-terminal to residue 120 have a more moderate role in influencing both aggregation and toxicity. A number of other properties of $\alpha$-synuclein may be important in disease pathogenesis, including ubiquitination (Anderson et al., 2006), phospholipid binding (Jo et al., 2000), tyrosine hydroxylase regulation (Perez et al., 2002), and chaperone function (Ostrerova et al., 1999). Additional investigations will be required to understand if these pathways directly influence the aggregation process of $\alpha$-synuclein or act independently to modulate neurotoxicity.

\section{References}

Anderson JP, Walker DE, Goldstein JM, de Laat R, Banducci K, Caccavello RJ, Barbour R, Huang J, Kling K, Lee M, Diep L, Keim PS, Shen X, Chataway T, Schlossmacher MG, Seubert P, Schenk D, Sinha S, Gai WP, Chilcote TJ (2006) Phosphorylation of Ser-129 is the dominant pathological modification of alpha-synuclein in familial and sporadic Lewy body disease. J Biol Chem 281:29739-29752.

Arrasate M, Mitra S, Schweitzer ES, Segal MR, Finkbeiner S (2004) Inclusion body formation reduces levels of mutant huntingtin and the risk of neuronal death. Nature 431:805-810.

Auluck PK, Chan HY, Trojanowski JQ, Lee VM, Bonini NM (2002) Chaperone suppression of alpha-synuclein toxicity in a Drosophila model for Parkinson's disease. Science 295:865-868.

Auluck PK, Meulener MC, Bonini NM (2005) Mechanisms of suppression of $\alpha$-synuclein neurotoxicity by geldanamycin in Drosophila. J Biol Chem 280:2873-2878.

Baba M, Nakajo S, Tu PH, Tomita T, Nakaya K, Lee VM, Trojanowski JQ, Iwatsubo T (1998) Aggregation of alpha-synuclein in Lewy bodies of sporadic Parkinson's disease and dementia with Lewy bodies. Am J Pathol 152:879-884.

Biere AL, Wood SJ, Wypych J, Steavenson S, Jiang Y, Anafi D, Jacobsen FW, Jarosinski MA, Wu GM, Louis JC, Martin F, Narhi LO, Citron M (2000) Parkinson's disease-associated alpha-synuclein is more fibrillogenic than 
beta- and gamma-synuclein and cannot cross-seed its homologs. J Biol Chem 275:34574-34579.

Bodles AM, Guthrie DJ, Greer B, Irvine GB (2001) Identification of the region of non-Abeta component (NAC) of Alzheimer's disease amyloid responsible for its aggregation and toxicity. J Neurochem 78:384-395.

Campbell BC, McLean CA, Culvenor JG, Gai WP, Blumbergs PC, Jakala P, Beyreuther K, Masters CL, Li QX (2001) The solubility of alphasynuclein in multiple system atrophy differs from that of dementia with Lewy bodies and Parkinson's disease. J Neurochem 76:87-96.

Chen L, Feany MB (2005) Alpha-synuclein phosphorylation controls neurotoxicity and inclusion formation in a Drosophila model of Parkinson disease. Nat Neurosci 8:657-663.

Clem RJ, Fechheimer M, Miller LK (1991) Prevention of apoptosis by a baculovirus gene during infection of insect cells. Science 254:1388-1390.

Conway KA, Harper JD, Lansbury PT (1998) Accelerated in vitro fibril formation by a mutant alpha-synuclein linked to early-onset Parkinson disease. Nat Med 4:1318-1320.

Conway KA, Lee SJ, Rochet JC, Ding TT, Williamson RE, Lansbury Jr PT (2000) Acceleration of oligomerization, not fibrillization, is a shared property of both alpha-synuclein mutations linked to early-onset Parkinson's disease: implications for pathogenesis and therapy. Proc Natl Acad Sci USA 97:571-576.

Cookson MR (2005) The biochemistry of Parkinson's disease. Annu Rev Biochem 74:29-52.

Crowther RA, Jakes R, Spillantini MG, Goedert M (1998) Synthetic filaments assembled from C-terminally truncated alpha-synuclein. FEBS Lett 436:309-312.

El-Agnaf OM, Jakes R, Curran MD, Wallace A (1998a) Effects of the mutations Ala30 to Pro and Ala53 to Thr on the physical and morphological properties of alpha-synuclein protein implicated in Parkinson's disease. FEBS Lett 440:67-70.

El-Agnaf OM, Jakes R, Curran MD, Middleton D, Ingenito R, Bianchi E, Pessi A, Neill D, Wallace A (1998b) Aggregates from mutant and wild-type alpha-synuclein proteins and NAC peptide induce apoptotic cell death in human neuroblastoma cells by formation of beta-sheet and amyloid-like filaments. FEBS Lett 440:71-75.

Feany MB, Bender WW (2000) A Drosophila model of Parkinson's disease. Nature 404:394-398.

Fujiwara H, Hasegawa M, Dohmae N, Kawashima A, Masliah E, Goldberg MS, Shen J, Takio K, Iwatsubo T (2002) alpha-Synuclein is phosphorylated in synucleinopathy lesions. Nat Cell Biol 4:160-164.

Giasson BI, Uryu K, Trojanowski JQ, Lee VM (1999) Mutant and wild type human alpha-synucleins assemble into elongated filaments with distinct morphologies in vitro. J Biol Chem 274:7619-7622.

Giasson BI, Duda JE, Murray IV, Chen Q, Souza JM, Hurtig HI, Ischiropoulos H, Trojanowski JQ, Lee VM (2000) Oxidative damage linked to neurodegeneration by selective alpha-synuclein nitration in synucleinopathy lesions. Science 290:985-989.

Giasson BI, Murray IV, Trojanowski JQ, Lee VM (2001) A hydrophobic stretch of 12 amino acid residues in the middle of alpha-synuclein is essential for filament assembly. J Biol Chem 276:2380-2386.

Giasson BI, Duda JE, Quinn SM, Zhang B, Trojanowski JQ, Lee VM (2002) Neuronal alpha-synucleinopathy with severe movement disorder in mice expressing A53T human alpha-synuclein. Neuron 34:521-533.

Glaser CB, Yamin G, Uversky VN, Fink AL (2005) Methionine oxidation, alpha-synuclein and Parkinson's disease. Biochim Biophys Acta 1703:157-169.

Greenbaum EA, Graves CL, Mishizen-Eberz AJ, Lupoli MA, Lynch DR, Englander SW, Axelsen PH, Giasson BI (2005) The E46K mutation in alpha-synuclein increases amyloid fibril formation. J Biol Chem 280:7800-7807.

Han H, Weinreb PH, Lansbury Jr PT (1995) The core Alzheimer's peptide NAC forms amyloid fibrils which seed and are seeded by beta-amyloid: is NAC a common trigger or target in neurodegenerative disease? Chem Biol 2:163-169.

Hay BA, Wassarman DA, Rubin GM (1995) Drosophila homologs of baculovirus inhibitor of apoptosis proteins function to block cell death. Cell 83:1253-1262.

Ibanez P, Bonnet AM, Debarges B, Lohmann E, Tison F, Pollak P, Agid Y, Durr A, Brice A (2004) Causal relation between alpha-synuclein gene duplication and familial Parkinson's disease. Lancet 364:1169-1171.

Jo E, McLaurin J, Yip CM, St George-Hyslop P, Fraser PE (2000) alpha-
Synuclein membrane interactions and lipid specificity. J Biol Chem 275:34328-34334.

Kruger R, Kuhn W, Muller T, Woitalla D, Graeber M, Kosel S, Przuntek H, Epplen JT, Schols L, Riess O (1998) Ala30Pro mutation in the gene encoding alpha-synuclein in Parkinson's disease. Nat Genet 18:106-108.

Lee MK, Stirling W, Xu Y, Xu X, Qui D, Mandir AS, Dawson TM, Copeland NG, Jenkins NA, Price DL (2002) Human alpha-synuclein-harboring familial Parkinson's disease-linked Ala-53 $\rightarrow$ Thr mutation causes neurodegenerative disease with alpha-synuclein aggregation in transgenic mice. Proc Natl Acad Sci USA 99:8968-8973.

Li H, Chaney S, Roberts IJ, Forte M, Hirsh J (2000) Ectopic G-protein expression in dopamine and serotonin neurons blocks cocaine sensitization in Drosophila melanogaster. Curr Biol 10:211-214.

Li HT, Du HN, Tang L, Hu J, Hu HY (2002) Structural transformation and aggregation of human alpha-synuclein in trifluoroethanol: non-amyloid component sequence is essential and beta-sheet formation is prerequisite to aggregation. Biopolymers 64:221-226.

Li J, Uversky VN, Fink AL (2002) Conformational behavior of human alpha-synuclein is modulated by familial Parkinson's disease point mutations A30P and A53T. Neurotoxicology 23:553-567.

Li W, West N, Colla E, Pletnikova O, Troncoso JC, Marsh L, Dawson TM, Jakala P, Hartmann T, Price DL, Lee MK (2005) Aggregation promoting C-terminal truncation of alpha-synuclein is a normal cellular process and is enhanced by the familial Parkinson's disease-linked mutations. Proc Natl Acad Sci USA 102:2162-2167.

Liu CW, Giasson BI, Lewis KA, Lee VM, Demartino GN, Thomas PJ (2005) A precipitating role for truncated alpha-synuclein and the proteasome in alpha-synuclein aggregation: implications for pathogenesis of Parkinson disease. J Biol Chem 280:22670-22678.

Mirzaei H, Schieler JL, Rochet JC, Regnier F (2006) Identification of rotenone-induced modifications in alpha-synuclein using affinity pulldown and tandem mass spectrometry. Anal Chem 78:2422-2431.

Moghal S, Rajput AH, D’Arcy C, Rajput R (1994) Prevalence of movement disorders in elderly community residents. Neuroepidemiology 13:175-178.

Murray IV, Giasson BI, Quinn SM, Koppaka V, Axelsen PH, Ischiropoulos H, Trojanowski JQ, Lee VM (2003) Role of alpha-synuclein carboxyterminus on fibril formation in vitro. Biochemistry 42:8530-8540.

Narhi L, Wood SJ, Steavenson S, Jiang Y, Wu GM, Anafi D, Kaufman SA, Martin F, Sitney K, Denis P, Louis JC, Wypych J, Biere AL, Citron M (1999) Both familial Parkinson's disease mutations accelerate alphasynuclein aggregation. J Biol Chem 274:9843-9846.

Neumann M, Kahle PJ, Giasson BI, Ozmen L, Borroni E, Spooren W, Muller V, Odoy S, Fujiwara H, Hasegawa M, Iwatsubo T, Trojanowski JQ, Kretzschmar HA, Haass C (2002) Misfolded proteinase K-resistant hyperphosphorylated alpha-synuclein in aged transgenic mice with locomotor deterioration and in human alpha-synucleinopathies. J Clin Invest 110:1429-1439.

Ostrerova N, Petrucelli L, Farrer M, Mehta N, Choi P, Hardy J, Wolozin B (1999) $\alpha$-Synuclein shares physical and functional homology with 143-3 proteins. J Neurosci 19:5782-5791.

Perez RG, Waymire JC, Lin E, Liu JJ, Guo F, Zigmond MJ (2002) A role for $\alpha$-synuclein in the regulation of dopamine biosynthesis. J Neurosci 22:3090-3099.

Pesah Y, Burgess H, Middlebrooks B, Ronningen K, Prosser J, Tirunagaru V, Zysk J, Mardon G (2005) Whole-mount analysis reveals normal numbers of dopaminergic neurons following misexpression of alphaSynuclein in Drosophila. Genesis 41:154-159.

Polymeropoulos MH, Lavedan C, Leroy E, Ide SE, Dehejia A, Dutra A, Pike B, Root H, Rubenstein J, Boyer R, Stenroos ES, Chandrasekharappa S, Athanassiadou A, Papapetropoulos T, Johnson WG, Lazzarini AM, Duvoisin RC, Di Iorio G, Golbe LI, Nussbaum RL (1997) Mutation in the alphasynuclein gene identified in families with Parkinson's disease. Science 276:2045-2047.

Saudou F, Finkbeiner S, Devys D, Greenberg ME (1998) Huntingtin acts in the nucleus to induce apoptosis but death does not correlate with the formation of intranuclear inclusions. Cell 95:55-66.

Serpell LC, Berriman J, Jakes R, Goedert M, Crowther RA (2000) Fiber diffraction of synthetic alpha-synuclein filaments shows amyloid-like crossbeta conformation. Proc Natl Acad Sci USA 97:4897-4902.

Singleton AB, Farrer M, Johnson J, Singleton A, Hague S, Kachergus J, Hulihan M, Peuralinna T, Dutra A, Nussbaum R, Lincoln S, Crawley A, Han- 
son M, Maraganore D, Adler C, Cookson MR, Muenter M, Baptista M, Miller D, Blancato J, et al. (2003) alpha-Synuclein locus triplication causes Parkinson's disease. Science 302:841.

Tofaris GK, Razzaq A, Ghetti B, Lilley KS, Spillantini MG (2003) Ubiquitination of alpha-synuclein in Lewy bodies is a pathological event not associated with impairment of proteasome function. J Biol Chem 278:44405-44411.

Tofaris GK, Garcia Reitbock P, Humby T, Lambourne SL, O'Connell M, Ghetti B, Gossage H, Emson PC, Wilkinson LS, Goedert M, Spillantini MG (2006) Pathological changes in dopaminergic nerve cells of the substantia nigra and olfactory bulb in mice transgenic for truncated human alpha-synuclein(1120): implications for Lewy body disorders. J Neurosci 26:3942-3950.

Trojanowski JQ, Lee VM (2000) "Fatal attractions" of proteins. A comprehensive hypothetical mechanism underlying Alzheimer's disease and other neurodegenerative disorders. Ann NY Acad Sci 924:62-67.

Volles MJ, Lansbury Jr PT (2003) Zeroing in on the pathogenic form of alpha-synuclein and its mechanism of neurotoxicity in Parkinson's disease. Biochemistry 42:7871-7878.

Wakamatsu M, Ishii A, Iwata S, Sakagami J, Ukai Y, Ono M, Kanbe D, Muramatsu SI, Kobayashi K, Iwatsubo T, Yoshimoto M (2007) Selective loss of nigral dopamine neurons induced by overexpression of truncated human alpha-synuclein in mice. Neurobiol Aging, in press.

Yamin G, Uversky VN, Fink AL (2003) Nitration inhibits fibrillation of human alpha-synuclein in vitro by formation of soluble oligomers. FEBS Lett 542:147-152.

Yang Y, Nishimura I, Imai Y, Takahashi R, Lu B (2003) Parkin suppresses dopaminergic neuron-selective neurotoxicity induced by Pael-R in Drosophila. Neuron 37:911-924.

Zarranz JJ, Alegre J, Gomez-Esteban JC, Lezcano E, Ros R, Ampuero I, Vidal L, Hoenicka J, Rodriguez O, Atares B, Llorens V, Gomez Tortosa E, del Ser T, Munoz DG, de Yebenes JG (2004) The new mutation, E46K, of alphasynuclein causes Parkinson and Lewy body dementia. Ann Neurol 55:164-173. 